\title{
Criminologie
}

\section{Une légalisation des drogues inscrite en promotion de la santé : les conditions}

\section{Line Beauchesne}

Volume 40, numéro 1, printemps 2007

Conduites addictives et crimes

URI : https://id.erudit.org/iderudit/016018ar

DOI : https://doi.org/10.7202/016018ar

Aller au sommaire du numéro

Éditeur(s)

Les Presses de l'Université de Montréal

ISSN

0316-0041 (imprimé)

1492-1367 (numérique)

Découvrir la revue

Citer cet article

Beauchesne, L. (2007). Une légalisation des drogues inscrite en promotion de la santé : les conditions. Criminologie, 40(1), 135-154.

https://doi.org/10.7202/016018ar
Résumé de l'article

La prohibition des drogues accroît les problèmes de santé publique et brime des fondements de la démocratie. Leur légalisation, soutenue par une réglementation appropriée, pourrait faciliter la mise en place d'une politique en matière de drogues, qui appuie un objectif de promotion de la santé dans un cadre démocratique. Dans cet article, nous identifierons les conditions auxquelles l'État doit souscrire afin que la légalisation des drogues soit un outil qui serve cet objectif dans le respect des citoyens. 


\title{
Une légalisation des drogues inscrite en promotion de la santé : les conditions
}

\author{
Line Beauchesne \\ Professeure titulaire \\ Département de criminologie \\ Université d'Ottawa \\ Line.Beauchesne@uottawa.ca
}

\begin{abstract}
RÉSUMÉ - La prohibition des drogues accroît les problèmes de santé publique et brime des fondements de la démocratie. Leur légalisation, soutenue par une réglementation appropriée, pourrait faciliter la mise en place d'une politique en matière de drogues, qui appuie un objectif de promotion de la santé dans un cadre démocratique. Dans cet article, nous identifierons les conditions auxquelles l'État doit souscrire afin que la légalisation des drogues soit un outil qui serve cet objectif dans le respect des citoyens.
\end{abstract}

ABSTRACT - Drug prohibition exacerbates public health problems and weakens the foundations of democracy. The legalisation of drugs, supported by appropriate regulation, could facilitate the introduction of a policy on drugs which would support a health promotion objective within the principles of democracy. In this article, I will discuss the conditions that the State needs to implement for the legalisation of drugs to become a tool that would serve the health promotion objective while respecting its citizens.

Dans une société libre et démocratique qui reconnaît fondamentalement, mais non exclusivement la primauté du droit comme source de règles normatives, et où la puissance publique doit le plus possible favoriser l'autonomie et conséquemment utiliser avec parcimonie les outils de contrainte, une politique publique sur les substances psychoactives doit s'articuler sur des principes directeurs respectant la vie, la santé, la sécurité et les droits et libertés de chaque individu qui, naturellement et légitimement, recherche son bien-être et son épanouissement, et a la capacité de reconnaître la présence, la différence et l'équivalence de l'autre. (Sénateur Nolin, président du Comité spécial du Sénat sur les drogues illicites, 2003: 129) 
La prohibition des drogues accroît les problèmes de santé publique et brime des fondements de la démocratie (Beauchesne, 2006a). Leur légalisation, soutenue par une réglementation appropriée, soit une légalisation contrôlée, pourrait faciliter la mise en place d'une politique en matière de drogues, qui souscrive à un objectif de promotion de la santé dans un cadre démocratique. Encore faut-il que l'État aille dans cette direction. Dans cet article, nous identifierons les conditions auxquelles l'État doit souscrire afin que la légalisation des drogues soit un outil qui serve cet objectif dans le respect des citoyens.

Dans un premier temps, nous clarifierons le concept de promotion de la santé et préciserons les prémisses nécessaires à ce que cet objectif s'inscrive dans un cadre démocratique. Par la suite, nous identifierons les obligations de l'État qui découlent de cette option politique en matière de drogues.

\section{Promotion de la santé et démocratie}

La santé ne signifie pas uniquement l'absence de maladie, et des politiques de santé ne se réduisent pas à un investissement dans le curatif. Promouvoir la santé est une orientation politique et des stratégies qui permettent d'augmenter la capacité des gens de faire des choix pour qu'ils puissent mieux gérer leur santé, soit leur bien-être physique et psychologique.

Autrefois, quand les maladies infectieuses étaient les affections les plus fréquentes et la principale cause de décès, la santé était définie comme l'absence de maladie. Cependant, vers le milieu du siècle, l'incidence d'un bon nombre de ces affections avait décru et la notion de santé prenait un nouveau sens plus large que le simple fait de ne pas être malade. Elle devenait un état de bien-être total sur les plans physique, mental et social. En 1974, une publication du gouvernement fédéral intitulée Nouvelle perspective de la santé des Canadiens faisait valoir que l'état de santé de la population était influencé par plusieurs facteurs: la biologie humaine, le mode de vie, l'organisation des soins de santé et l'environnement physique et social dans lequel les gens vivent. Cette image des facteurs qui contribuent à la santé justifiait l'idée d'élaborer des politiques et des mesures qui s'inscriraient dans un cadre plus vaste.

Nous travaillons maintenant dans l'idée que la santé est un aspect de la vie courante, une dimension essentielle de notre qualité de vie. Envisagée sous cet angle, la qualité de vie suppose la possibilité de faire des choix et d'avoir 
un certain plaisir à vivre. La santé paraît alors comme une ressource qui permet aux gens d'exploiter leur environnement et même de le modifier. Considérer la santé sous ce jour, c'est admettre la liberté de choix et souligner le rôle des individus et des collectivités lorsqu'il s'agit de définir le sens que la santé peut avoir pour eux. (Santé et bien-être social Canada, 1986: 3)

Cette approche canadienne en matière de promotion de la santé s'est renforcée à la première Conférence internationale pour la promotion de la santé tenue à Ottawa. À cette occasion, fut adoptée, le 21 novembre 1986, une charte sur un projet de promotion de la santé désignée sous le vocable de Charte d'Ottawa.

La promotion de la santé a pour but de donner aux individus davantage de maîtrise de leur propre santé et davantage de moyens pour l'améliorer. Pour parvenir à un état de complet bien-être physique, mental et social, l'individu, ou le groupe, doit pouvoir identifier et réaliser ses ambitions, satisfaire ses besoins et évoluer avec son milieu ou s'y adapter. La santé est donc perçue comme une ressource de la vie quotidienne, et non comme le but de la vie; c'est un concept positif mettant l'accent sur les ressources sociales et personnelles et sur les capacités physiques. La promotion de la santé ne relève donc pas seulement du secteur de la santé: elle ne se borne pas seulement à préconiser l'adoption de modes de vie qui favorisent la bonne santé; son ambition est le bien-être complet de l'individu. (OMS - Organisation mondiale de la santé, 1986)

À cette fin, la charte détermine certaines conditions nécessaires pour servir correctement cet objectif:

- Se loger;

- Accéder à l'éducation;

- Se nourrir convenablement;

- Disposer d'un certain revenu;

- Bénéficier d'un écosystème stable;

- Compter sur un apport durable de ressources;

- Avoir droit à la justice sociale et à un traitement équitable.

Tels sont les préalables indispensables à toute amélioration de la santé (OMS, 1986).

Nous bénéficions par ce projet de promotion de la santé d'un outil précieux pour réfléchir à une politique plus appropriée en matière de drogues.

Tout d'abord, ce projet s'articule sur le respect de la citoyenneté en prônant le libre choix des individus dans la définition de leur bien-être. 
Ce dernier élément signifie qu'il faut sortir d'une vision morale des objectifs d'une politique publique en matière de drogues qui ne viserait qu'à réduire l'accès aux drogues (légales ou illégales), indépendamment de leur gestion par les individus.

Ensuite, ce projet permet de définir cette politique en dehors d'une vision strictement médicale pour considérer l'ensemble des déterminants de la santé, soit le produit, la relation de la personne avec le produit et l'environnement dans lequel elle vit pour identifier les meilleures stratégies visant à réduire des usages problématiques ou inappropriés. Le produit n'est plus isolé de la signification que la personne donne à sa consommation, ou encore de son rôle adaptatif à l'environnement.

Qui oserait affirmer que l'alcoolisme massif des populations ouvrières du $\mathrm{XIX}^{\mathrm{e}}$ siècle ou la toxicomanie récurrente de certaines couches de population aujourd'hui encore particulièrement défavorisées - songeons un instant aux jeunes des banlieues des métropoles industrielles ou aux populations des cités mouroirs des pays du Tiers Monde subissant aujourd'hui l'assaut des drogues illicites - serait le fruit d'un choix libre, conscient et responsable? (Van der Smissen et Picard, 1990: 216)

Quand les conditions d'une vie décente ne sont pas réunies, il est clair que la consommation de drogues revêt une signification particulière et risque davantage de devenir problématique. Ces problèmes ne peuvent être réduits à de «mauvais» choix individuels d'où on déduirait qu'il faut resserrer le cadre légal. Constater que des jeunes Autochtones reniflent de la colle n'ouvre pas un débat sur la colle et sa réglementation, mais sur le rôle de l'État à l'égard des communautés autochtones et sur leurs conditions de vie. Négliger ce débat permet trop aisément à l'État de nier ses responsabilités pour améliorer les conditions socioéconomiques, le premier défi que reconnaît avec justesse ce projet de promotion de la santé.

Ces éléments étant clarifiés, il découle qu'une politique en matière de drogues doit abolir la séparation juridique entre les drogues du Nord (légales) et les drogues du Sud (illégales) pour réfléchir à une politique qui les englobe dans leur ensemble, avec cohérence et équité, des antidépresseurs à l'héroöne, du cannabis à l'alcool.

Enfin, une des stratégies privilégiée pour la mise en œuvre de ce projet est le «renforcement de l'action communautaire» (OMS, 1986). Cette stratégie repose entre autres sur la décentralisation des décisions quant aux pratiques à implanter dans les communautés de manière à 
mieux respecter les citoyens. À cet égard, il y a beaucoup à apprendre des pays qui, même dans le cadre de la prohibition, tels les Pays-Bas ou la Suisse, ont donné la possibilité aux communautés locales d'adapter certaines politiques gouvernementales en matière de drogues à leurs problèmes spécifiques (Collin, 2002; Keiser, 2001).

On se rend compte qu'une décentralisation des structures décisionnelles dans l'implantation des pratiques facilite les débats qui permettent à la population d'être mieux informée et respecte davantage l'intégration culturelle de nouvelles voies. Par exemple, aux Pays-Bas, les différentes communautés locales n'ont pas fait les mêmes choix sur l'ouverture et même la réglementation des coffee-shops (Garretsen, 2003). Ou encore, en Suisse, les centres de prescription d'héroïne présents dans certains cantons furent mis en place et maintenus à la suite d'une consultation gouvernementale des citoyens (Büchli, 2002). Le soutien communautaire de ces pratiques nouvelles permet aux intervenants de travailler dans un environnement plus accueillant et les rend moins vulnérables aux critiques à la moindre erreur d'opération. Décentraliser le processus d'implantation de pratiques nouvelles sur un plan plus local laisse le temps à leur intégration culturelle, évitant que la population ne se sente agressée par l'incompréhension des objectifs recherchés par ces pratiques.

Quel est alors le rôle de l'État dans une politique en matière de drogues qui respecte ces assises? La création d'un cadre juridique qui norme avec cohérence la problématique des drogues de manière à privilégier un accès sécuritaire aux produits et à réduire les méfaits potentiels de leur consommation.

\section{Les obligations de l'État en promotion de la santé}

\section{La réduction des usages à risques}

Comme il relève de la responsabilité de l'État que la consommation des aliments ou d'autres produits soit la plus sécuritaire possible pour les citoyens, il est également de sa responsabilité d'assurer un contexte sécuritaire de consommation en matière de drogues. Pour ce faire, certains contrôles relevant d'un volet de réduction des usages à risques sont essentiels. 


\section{Contrôle de leur composition}

L'État doit implanter une réglementation qui assure des contrôles adéquats appropriés à la qualité des produits. Cette réglementation, si nécessaire, peut mener à modifier certaines composantes, conditions de culture ou de fabrication d'une drogue pour en diminuer la nocivité. En ce qui a trait aux drogues qui sont actuellement illicites, il est certain que le contrôle de leur qualité et de leur concentration pour les rendre plus sécuritaires ferait en sorte que ces drogues n'auraient plus grandchose à voir avec la composition de celles qui circulent actuellement sur le marché noir. Lorsqu'on a mis fin à la prohibition de l'alcool, on n'a pas légalisé l'alcool frelaté. L'alcool frelaté est disparu avec l'implantation d'une légalisation contrôlée de cette drogue. De même, lors de la prohibition de l'alcool, ce n'était pas de la bière à $0.5 \%$ d'alcool qui était trafiquée, mais de l'alcool à haute concentration.

Durant la prohibition de l'alcool, la consommation s'est moulée aux nécessités du marché noir. Il était plus profitable et moins cher de distribuer des alcools distillés (gin, vodka, whisky ou rhum) que la bière. [...] La production et l'entreposage de la bière demandent d'énormes contenants, plusieurs barils, d'énormes camions et un investissement substantiel en équipement. [...] Parce que le taux d'alcool était le principal déterminant du prix, les alcools distillés rapportaient plus que la bière et aussi se camouflaient et se transportaient plus aisément. De plus, les alcools distillés pouvaient se conserver indéfiniment, tandis que la bière pouvait se gâter rapidement. L'embouteillage à grande échelle de la bière et sa réfrigération se sont développés dans les années 1930, après la prohibition. (Levine et Reinarman, 2004: 7, notre traduction)

À la suite de la prohibition, les monopoles gouvernementaux sur cette drogue se sont multipliés. En Amérique du Nord, le Québec, seule province à ne pas avoir adhéré à la prohibition lors d'un référendum au Canada en 1898, ouvrit le bal en 1921 en créant la Commission des liqueurs. Son mandat initial était «de favoriser la consommation modérée de boissons alcooliques, de qualité dûment vérifiée, vendues à un prix raisonnable, et ce, dans un cadre d'exploitation contrôlée» (Fortin, 2006: 1).

Ce geste du gouvernement québécois traduit bel et bien une préoccupation sanitaire plutôt qu'économique. Ainsi, dès sa création, la Commission des liqueurs encourage la consommation du vin au détriment des boissons fortes en tant que mesure d'éducation à la modération [...] Au cours des 
années 1920, le Québec va devenir un haut lieu de l'alcootourisme - à l'instar du narcotourisme que connaissent de nos jours les Pays-Bas en raison d'un accès libéralisé au cannabis - et des entrepreneurs locaux s'enrichiront avec le trafic continental de l'alcool. Fait d'importance, le choix historique d'un modèle antiprohibitionniste n'a pas fait du Québec un champion de la consommation d'alcool, beaucoup s'en faut: encore maintenant, la consommation per capita des Québécois est moindre que celle de leurs vis-à-vis canadiens-anglais ou américains. D'ailleurs, lorsque la prohibition de l'alcool s'avère un échec chez nos voisins anglo-saxons, quelques années plus tard, tous les yeux se tournent vers le modèle québécois dont la notoriété attire des observateurs de partout dans le monde. (Brisson, 2000: 8-9)

De 1933 à 1935, au moins quinze États américains «adoptent une structure de monopole d'État semblable à celle du Québec» (Fortin, 2006: 1).

Cette orientation vers la promotion de la santé ne s'est pas faite sans mal. D'un côté, il y avait les partisans d'une déréglementation de cette drogue, appuyés par l'industrie de l'alcool et les publicitaires, et de l'autre, des scientifiques et des professionnels de la santé qui demandaient que l'État joue son rôle de créer un environnement sécuritaire pour la consommation, vu les méfaits que certains usages de cette drogue peuvent générer (Babor et al., 2003; Room, 2004).

L'implantation d'une légalisation contrôlée a permis la fabrication de produits alcoolisés avec divers taux de concentration d'alcool et même des réglementations différentes sur la circulation et la taxation des produits selon leur concentration d'alcool. Enfin, les modes de consommation d'alcool sous la prohibition étaient plus durs, parce que concentrés dans des temps et des espaces délimités. Le retour à la légalisation a permis d'apprendre à gérer l'alcool autrement. Il en va de même pour les changements qui affecteraient le marché illégal actuel; les produits frelatés disparaîtraient et des formes de consommation plus douces de ces drogues pourraient voir le jour dans un contexte où il serait plus aisé d'apprendre à les consommer de manière sécuritaire. Mais pour que cela se produise, il est nécessaire que les contrôles gouvernementaux soient adéquats.

Prenons le cas du tabac. Il est clair que les compagnies qui fabriquent des produits avec cette drogue, comme toute compagnie, recherchent l'augmentation de leurs profits. Pour ce faire, elles ont entre autres modifié le produit de manière à en augmenter la pharmacodépendance 
(<www.library.ucsf.edu/tobacco/> ; Fenn et Schroeter, 2004; LeGresley et al., 2005; Muggli et al., 2004a; 2004b; 2004c). Par exemple, on augmente les doses de nicotine dans les produits par différentes manipulations chimiques et même génétiques. Ou encore, on ajoute à la cigarette certains produits pour accroître la rétention de la nicotine dans les poumons (ajout d'ammoniaque), ou encore l'absorption de nicotine par l'organisme (avec les cigarettes au menthol ou encore l'ajout d'acide «levulinic» dans les cigarettes «légères» et «ultra-légères») (Garten et Falkner, 2004; Keithly et al., 2005). Une bonne partie de ces additifs se retrouvent également dans la fumée secondaire (Muggli et al., 2003). Le gouvernement a un rôle à jouer pour contrer cette action des compagnies en contrôlant mieux la fabrication de ces produits.

Idéalement, une réglementation appropriée de l'industrie du tabac devrait encadrer les six éléments suivants:

- Les caractéristiques des produits, incluant les ingrédients qui entrent dans leur processus de fabrication;

- L'information donnée aux consommateurs sur les produits, tant sur les paquets que de manière plus globale, incluant la publicité et la mise en marché des produits, et l'assurance que cette information inclut les risques pour la santé et les moyens de cesser cette habitude;

- Le développement et la distribution de produits moins nocifs et pharmacodépendants;

- Le contrôle des prix en tant que moyen d'en décourager l'usage, particulièrement chez les jeunes, ou encore la variation des prix en fonction de la nocivité des produits pour encourager la consommation de produits moins nocifs;

- Le contrôle des lieux de vente de ces produits par des licences et l'implantation d'une réglementation à suivre pour opérer;

- La création d'une agence de contrôle responsable de l'application adéquate de la réglementation et du suivi en cas d'infractions. (Liberman, 2003: 465-466, notre traduction)

Le tabac est une drogue et il ne s'agit pas d'en promouvoir l'usage. $\mathrm{Au}$ contraire. Toute la réglementation et le contrôle doivent s'inscrire dans un objectif de promotion de la santé visant à réduire l'usage de cette drogue et les méfaits chez ceux qui en consomment directement ou indirectement. C'est pourquoi, idéalement, les compagnies de tabac, qui sont gérées suivant une logique de rentabilité amenant à augmenter la pharmacodépendance des produits et à développer des stratégies pour accroître le nombre de consommateurs, devraient être démantelées au 
profit d'un nouveau système de licences basé sur des priorités en promotion de la santé, avec des stratégies de réduction des méfaits tant dans la mise en marché que dans la fabrication des produits. Ainsi, ces produits pourraient être de maintenance, pour ceux qui ne sont pas prêts à arrêter de consommer, ou encore faciliter la diminution progressive des doses de produits nocifs et créant une pharmacodépendance, quand la personne veut utiliser cette méthode pour réduire sa consommation, ou encore, des substituts du tabac quand il ne reste que la dépendance buccale. Jamais les compagnies de tabac ne répondront à cette logique de fabrication des produits, qui vise à diminuer le nombre de consommateurs de cette drogue et les méfaits sur la santé (Hatsukami et al., 2001 ; Parascandola, 2005 ; Warner, 2002). Mais les gouvernements qui trouvent dans la taxation des produits du tabac un important revenu, sont-ils intéressés par une nationalisation de ces produits afin d'en permettre un meilleur contrôle pour la promotion de la santé?

La question soulevée ici est fondamentale, que ce soit avec le tabac, drogue gérée par le privé, ou même avec l'alcool, drogue gérée par l'État au Canada.

En effet, au début des années 1970, en fonction des politiques passées, l'alcool s'inscrit dans les politiques publiques avec une priorité claire en promotion de la santé. Cela ne s'est pas fait sans mal car, d'un côté, il y avait les partisans d'une déréglementation de cette drogue, appuyés par l'industrie de l'alcool et les publicitaires, et de l'autre, des scientifiques et des professionnels de la santé qui demandaient que l'État joue son rôle de créer un environnement sécuritaire pour la consommation, vu les méfaits que certains usages de cette drogue peuvent générer (Babor et al., 2003; Room, 2004).

Au Canada, cette priorité de la promotion de la santé s'est traduite dans la plupart des provinces par l'instauration d'une Régie des alcools. Mais depuis le milieu des années 1990, cette priorité accordée à la promotion de la santé s'affaiblit au profit d'une gestion de cette drogue comme dans le marché privé, soit la promotion de sa consommation pour la maximisation des profits. Comme on peut le voir dans le tableau suivant, cette augmentation du volume des ventes se traduit en dividendes gouvernementaux importants. 
TABLEA U 1

Total du bénéfice net des régies des alcools provinciales et territoriales, et recettes des administrations provenant directement du contrôle et de la vente des boissons alcoolisées en milliers de dollars

(Ne comprend pas les taxes générales de vente au détail perçues par la plupart des provinces)

\begin{tabular}{|c|c|c|c|c|c|c|c|c|c|c|}
\hline $\begin{array}{l}\text { Province ou } \\
\text { territoire }\end{array}$ & 1995 & 1996 & 1997 & 1998 & 1999 & 2000 & 2001 & 2002 & 2003 & 2004 \\
\hline $\begin{array}{l}\text { Terre-Neuve- } \\
\text { et-Labrador }\end{array}$ & 78779 & 77483 & 76087 & 85763 & 87185 & 92830 & 96141 & 93118 & 92270 & 100038 \\
\hline $\begin{array}{l}\text { Île-du-Prince- } \\
\text { Édouard }\end{array}$ & 18524 & 19267 & 18700 & 19012 & 10134 & 20408 & 20482 & 21211 & 21567 & 22670 \\
\hline $\begin{array}{l}\text { Nouvelle- } \\
\text { Écosse }\end{array}$ & 118654 & 119990 & 122036 & 125510 & 136410 & 141526 & 144638 & 151434 & 161515 & 171621 \\
\hline $\begin{array}{l}\text { Nouveau- } \\
\text { Brunswick }\end{array}$ & 86502 & 88037 & 88649 & 88600 & 95672 & 103972 & 106686 & 121725 & 113694 & 121904 \\
\hline Québec & 443407 & 476977 & 494652 & 507203 & 542986 & 574704 & 617786 & 628717 & 695107 & 714818 \\
\hline Ontario & 1159414 & 1196405 & 1221845 & 1250561 & 1328898 & 1404552 & 1400385 & 1452384 & 1402302 & 1534521 \\
\hline Manitoba & 143954 & 144373 & 145875 & 150623 & 152919 & 157505 & 159839 & 164665 & 167647 & 177586 \\
\hline Saskatchewan & 123101 & 122324 & 121652 & 125712 & 129571 & 129970 & 127896 & 124428 & 126425 & 134544 \\
\hline Alberta & 436256 & 420016 & 430172 & 456154 & 474042 & 461219 & 476310 & 499449 & 541312 & 556608 \\
\hline $\begin{array}{l}\text { Colombie- } \\
\text { Britannique }\end{array}$ & 573917 & 572926 & 592589 & 612045 & 614056 & 615496 & 649026 & 643.373 & 660424 & 733749 \\
\hline $\begin{array}{l}\text { Territoire du } \\
\text { Yukon }\end{array}$ & 6555 & 7656 & 7782 & 7903 & 7027 & 6978 & 7088 & 7062 & 6889 & 7915 \\
\hline $\begin{array}{l}\text { Territoires du } \\
\text { Nord-Ouest }\end{array}$ & 15418 & 16380 & 16253 & 16470 & 16647 & 16074 & 16830 & 18944 & 19819 & 20420 \\
\hline Nunavut & ---.- & ----- & ----- & ---- & ---.- & 1799 & 2191 & 2176 & 1813 & 1923 \\
\hline CANADA & 3204481 & 3261834 & 3336292 & 3445556 & 3604548 & 3727033 & 3825298 & 3928696 & 4010885 & 4298317 \\
\hline
\end{tabular}

Source: Statistique Canada, $n^{\circ}$ 63-202 au catalogue 1.

Ce changement de tendance s'est traduit par une augmentation des ventes qui avaient diminué à la suite des mesures plus élaborées de contrôle et de prévention appliquées au cours des années 1970. En 1974, les ventes moyennes de boissons alcoolisées par habitant au Canada étaient de 134 litres. Ce taux a baissé jusqu'en 1996 pour atteindre 99,5 litres. Puis il remonte pour se situer, en 2004, à 107,2 litres (Santé Canada et al., 2005).

Cette recherche gouvernementale de profits avec l'alcool, à l'instar du marché privé du tabac ${ }^{1}$, montre l'importance d'ouvrir le débat sur le fait que les drogues ne sont pas des produits comme les autres et que l'on doit rappeler à l'État ses obligations en matière de promotion de la santé. C'est ce qui amène à poser la question de l'objectif de la taxation d'une drogue.

1. On peut inclure ici les compagnies pharmaceutiques (Angell, 2004; Fisher, 2003; Mather, 2005; Robinson, 2001 ; St-Onge, 2004; Tracy, 2004). 


\section{La taxation}

Un des principaux outils utilisés par l'État pour réguler certains usages d'alcool ou de tabac est la taxation. De nombreuses études montrent que des prix plus élevés réduisent l'incidence de la consommation de ces drogues, particulièrement chez les plus jeunes et les gros consommateurs (Adrian et al., 2001; Grube et Stewart, 2004). Demeure la question du taux de taxation. L'État cherche généralement à imposer des taxes assez élevées pour en tirer un revenu le plus intéressant possible, mais cependant pas trop prohibitives, qui affecteraient négativement les ventes et favoriseraient un marché noir plus important ou encore un accroissement des pratiques de fabrication artisanale (Jazairi, 1994; Macdonald, 1999). Le modèle prédominant de taxation des produits alcoolisés est de moduler la taxe en fonction de la concentration d'alcool, ce qui est logique dans une perspective de santé publique, les produits moins alcoolisés étant moins taxés que les produits plus alcoolisés.

Sur quels principes instaurer ces taxes pour l'ensemble des drogues de manière à ce que le gouvernement en reçoive les dividendes tout en respectant l'objectif prioritaire de promotion de la santé?

Comme dans le cas de l'alcool, les drogues devraient être taxées proportionnellement aux torts potentiels suite à l'évaluation scientifique de leurs effets selon les modes de consommation et leur concentration. Cela pourrait, par exemple, signifier une taxation plus élevée du café et des colas que des tisanes de coca, de la cigarette de tabac plus que sur celle de cannabis ou d'opium, etc., selon, bien sûr, les concentrations et les modes de consommation de chaque substance. Ce mode de taxation proportionnel aux risques pour la santé du consommateur représentés par le produit a l'avantage d'être pédagogique en rendant visibles les usages plus doux et plus durs de drogues, de la même manière qu'avec l'alcool. Il aiderait également à faire comprendre des restrictions plus grandes et des règles plus strictes pour certains usages durs de drogues. Enfin, il faciliterait la mise en place de modèles de prévention qui feraient écho aux politiques en place.

Les revenus générés par ces taxes devraient être attribués à des projets concrets et limités dans le temps, à la suite d'un vote démocratique. Par exemple, la construction de centres de traitement de toxicomanies, l'implantation de programmes de prévention des usages problématiques de drogues, voire la dépollution de rivières ou tout autre 
projet susceptible d'améliorer la qualité de vie. Il doit s'agir de projets ponctuels pour que l'État n'ait pas intérêt à encourager la consommation de drogues pour récolter des taxes générant des revenus espérés pour la gestion des dépenses courantes (Mitchell, 1990).

Car là est le nœud du problème. Quand le gouvernement priorise la récolte de dividendes sur la promotion de la santé, les contrôles sur les drogues s'atténuent et font place à une promotion des produits sur le modèle du marché privé. C'est pourquoi il faut nuancer un discours trop souvent entendu chez certains partisans de la légalisation des drogues actuellement illicites, particulièrement du cannabis, à savoir qu'il serait plus rentable pour le gouvernement de légaliser les drogues. Cela n'est pas si sûr, considérant l'ensemble des mesures à mettre en place pour promouvoir la santé. S'il est normal qu'une certaine taxation des drogues existe pour diminuer certains types de consommation, une bonne taxation inscrite dans un projet de promotion de la santé amène nécessairement une stagnation des dividendes gouvernementaux sur les drogues. Le plafonnement des revenus des drogues va de pair avec le plafonnement de leur consommation. Ce débat peut s'ouvrir dès maintenant avec les drogues légales.

\section{Contrôle de leur mise en marché}

Il découle de cette taxation inscrite en promotion de la santé que l'État doit élaborer une réglementation qui assure que la commercialisation d'une drogue ne signifie pas sa promotion, mais une information adéquate au consommateur, entre autres, par des restrictions publicitaires et un étiquetage précis.

Marques et publicités sont interdites et remplacées par toutes les informations nécessaires à un choix librement consenti: la provenance, la méthode de fabrication, le détail des principes actifs, les risques sanitaires répertoriés, les circonstances dans lesquelles s'abstenir de consommer.

Il s'agit donc simultanément de casser les circuits de promotion, de réduire l'offre et de respecter la demande. Respecter la demande ne signifie pas renoncer à influencer le demandeur; on doit toujours l'inciter à faire preuve de responsabilité: en le dissuadant notamment des usages durs et en faisant la promotion des usages doux (sniffer plutôt que se piquer ou fumer; espacer les prises; consommer une substance et non plusieurs; user des substances les moins nocives). Les recommandations doivent être appropriées à l'état du demandeur et selon les risques liés à l'usage, à partir 
d'informations détaillées sur la composition des drogues. (Colle, 2000: 151-152)

À cet égard, le gouvernement contrôle mal l'information distribuée par le marché privé des drogues. Par exemple, la publicité frauduleuse des compagnies de tabac sur les cigarettes supposément «légères», les taux de nicotine et de goudron indiqués sur les paquets de cigarettes qui ne correspondent pas à l'absorption réelle de ces produits par le consommateur, etc., n'amènent pas de sanctions gouvernementales à l'égard de ces compagnies.

Les recherches ont depuis longtemps dénoncé le fait que les appareils [des compagnies] pour mesurer la nicotine et le goudron ne reflètent pas l'absorption réelle de ces produits par le fumeur. Les fumeurs [...] compensent la présence du filtre et du taux plus faible de nicotine et de goudron en inhalant plus profondément, plus fréquemment, terminant la cigarette jusqu'au filtre, ou fumant davantage. De plus, les fumeurs, contrairement aux appareils mesurant la nicotine et le goudron, bloquent les trous de ventilation du filtre lorsqu'ils tiennent leur cigarette. (Warner, 2002: S65, notre traduction)

Là aussi le gouvernement peut corriger le tir dès maintenant pour que le citoyen soit mieux informé sur cette drogue, en interdisant la publicité frauduleuse et en corrigeant l'étiquetage.

\section{Contrôle des lieux de distribution}

L'État doit également implanter une réglementation sur la distribution de la drogue. Selon les cas, la disponibilité des drogues peut être restreinte à certains lieux licenciés et à certaines heures, de même que l'on peut en limiter l'accès aux mineurs. Les drogues illicites sont actuellement en vente partout et aisément accessibles aux mineurs. Délivrer des licences à des lieux spécifiques de vente de drogues (tabac, alcool et autres) avec une réglementation soumise à des inspections d'une régie qui délivre ces licences pour en vérifier l'application est nécessaire (Haden, 2004; Stockwell, 1997).

\section{Prévention}

Enfin, le gouvernement doit rendre disponible les fonds nécessaires à la mise en place de stratégies de prévention permettant d'informer le citoyen des bienfaits et des méfaits de l'usage selon les produits, les 
modes de consommation et les contextes d'utilisation. Il ne sert à rien de faire semblant que les sollicitations environnantes n'existent pas. Il faut apprendre à vivre parmi ces produits.

À l'heure actuelle, il n'est pas étonnant que l'on ait fait de la prévention des usages problématiques de drogues un monde à part où les citoyens se sentent démunis, particulièrement en ce qui a trait aux drogues illicites. Dans le discours dominant, retransmis par les médias et ailleurs, les drogues illicites sont comparées à une sorte d'épidémie qui peut «contaminer» notre jeunesse sans que l'on ne puisse rien faire d'autre que lui éviter à tout prix tout contact avec ces drogues. Cette information omet de distinguer l'essai d'une drogue, sa gestion adéquate et la dépendance qui peut en découler, dépendance liée à une interaction entre la personne, le produit et son environnement.

La prise en compte de cette interaction est centrale en prévention. Il ne s'agit pas uniquement de décrire les produits et de miser sur la peur en montrant les risques de la consommation. En prévention, miser uniquement sur les risques des drogues plutôt que sur ce qui mène à la dépendance crée un message très ambigu, particulièrement chez les jeunes, qui doivent faire face, d'un côté, au risque déviant - l'usage de drogues - et de l'autre, au risque valorisé - les autres conduites à risques, sportives ou autres, promues tant dans les activités que par les héros proposés actuellement aux jeunes comme modèles d'identification:

De nouvelles formes de loisirs - largement relayées par la publicité - ne cessent de voir le jour, qui renouvellent perpétuellement ce jeu délibéré du risque: saut à l'élastique, escalades extrêmes, exploits de l'impossible, raidsaventure, free-ride et ski hors-piste, courses au bout du monde... Évasions jubilatoires réelles ou par procuration, mais recherchées, semble-t-il, en contrepoint d'une vie tellement tranquillisée, pacifiée et sécurisée qu'elle en est vidée d'imprévu, privée d'émotion et dépourvue d'attrait [...]

Chaque individu se trouve face à ce même dilemme où la rationalité et l'imaginaire ont du mal à se rejoindre et à s'allier. La prise de risque est une manière d'explorer et d'interroger le monde sur la marge de liberté qu'il nous laisse. Mais, en même temps, la maîtrise des risques est un des critères de détermination du niveau d'autonomie de l'individu. Les risques et leur apprivoisement sont donc au cœur des missions de l'éducation. (Morel, 2000: 57-58)

Les parents devraient être les premiers à assumer ce rôle en prévention d'usages problématiques de drogues auprès des jeunes (Beauchesne, 2005). Pour ce faire, la responsabilité du gouvernement est de fournir 
un cadre de réglementations qui facilite ce travail de prévention, et une mise en marché des produits qui ne contrecarre pas les messages de prévention par la promotion de l'usage des drogues.

En fait, dès maintenant, le gouvernement peut agir pour modifier tant le discours justifiant la prohibition de certaines drogues, qui dramatise outre mesure la dangerosité en soi des produits, comme la publicité trompeuse, qui banalise des comportements présentant certains risques.

\section{La réduction des méfaits}

Un volet de réduction des conséquences négatives liées à un usage problématique doit également être soutenu par une politique adéquate en matière de drogues qui s'inscrive en promotion de la santé. À cette fin, l'État doit mettre en place une gamme de soins variés pour répondre aux besoins des usagers qui connaissent des problèmes.

En cette période de restrictions budgétaires, circule un discours à l'effet que les usagers de drogues auraient pu ne pas avoir cette habitude et n'auraient donc pas droit aux mêmes soins que les «bons citoyens». Bien sûr, idéalement, on ne devrait pas trop fumer, pas trop boire de café, toujours bien manger, éviter le stress, avoir de bonnes habitudes de vie. Mais vivre, c'est également assumer un certain nombre de risques pour répondre à nos aspirations ou pour s'adapter aux événements de notre vie. Cela vaut aussi bien pour la personne qui se casse la jambe en ski et qui aurait pu éviter cette activité que pour celles qui ont des problèmes de poids à cause de leur alimentation et qui auraient pu toujours prendre le temps de bien manger et faire de bons choix, ou qui ont des problèmes cardiaques à cause du surmenage au travail, etc. Nos habitudes de vie sont non seulement le reflet de décisions pesées rationnellement, mais le reflet de nos désirs, de nos objectifs, de nos interactions sociales et familiales, de nos conditions de vie qui nous ont amenés à faire certains choix, parfois heureux, parfois malheureux. En fait, ce n'est pas le risque que l'on choisit, mais des objectifs ou habitudes qui comportent un certain nombre de risques et plus le désir est fort, plus on a tendance à minimiser les risques. Il en va de même pour l'usage de drogues.

On ne traverse pas la rue pour prendre un risque, mais pour se rendre quelque part. [...] Qu'il s'agisse de tabac ou de plongée sous-marine, de journalisme ou de travail dans la mine, le choix individuel se fait dans un 
cadre qui délimite, dans un groupe donné, le risque acceptable du risque inacceptable.

[...] Il faut se rappeler que, du soulagement de la douleur à la recherche du plaisir, de l'expansion de la conscience à la performance, la consommation de drogues obéit à bien d'autres motivations; le risque est souvent minimisé, ce qui ne veut pas dire qu'il est recherché. (Coppel, 2002: 130-131)

Une habitude qui est d'abord apparue comme une solution pour se détendre, se donner plus d'énergie au travail, faciliter les interactions sociales et amoureuses, peut se révéler néfaste à la longue. Parfois, également, une habitude de consommation de drogues échappe au contrôle de la personne, ne répond plus à ses attentes, tout en demeurant une habitude qu'elle ne sait pas encore remplacer, pour toutes sortes de raisons. Quand une personne en est là, il faut qu'elle puisse trouver de l'aide si elle en ressent le besoin.

Toute personne qui s'intéresse de près ou de loin au problème de la toxicomanie est bien consciente que la vraie question est: pourquoi un toxicomane se drogue-t-il? Et non: quel produit prend-il? [...]

Il est singulier de constater qu'en prétendant s'attaquer aux «vrais problèmes» les tenants de la prohibition concentrent l'essentiel de leurs attaques - et de leur budget - sur l'offre de stupéfiants et les moyens de l'endiguer, comme si la question de la demande de drogue n'était qu'accessoire. (Van der Smissen et Picard, 1990 : 223)

Pour assurer cette aide, l'État doit consentir les fonds nécessaires pour la formation des intervenants afin qu'ils puissent répondre à la multiplicité des problèmes. Cela va des soins médicaux à l'accompagnement thérapeutique, jusqu'aux interventions pour modifier l'environnement de certaines communautés ou milieux de vie. Il ne faut pas abandonner les problèmes de consommation au domaine strictement médical, car les motifs de consommation vont bien au-delà du produit. De plus, une formation devrait être requise pour tous les vendeurs de drogues (tabac, alcool, produits pharmaceutiques et autres drogues) afin d'assurer une prévention et une intervention adéquates auprès du consommateur.

Enfin, particulièrement pour les drogues actuellement illégales, il faudra consacrer des fonds à la recherche pour parfaire nos connaissances sur les produits, les risques liés aux différents usages durs et doux, les causes des différences dans les modes de consommation selon les populations. Ce savoir permettra d'améliorer continuellement la réglementation, la prévention et les soins. 


\section{Conclusion}

Une politique citoyenne en matière de drogues

La citoyenneté consiste $[\ldots]$ en une pratique de ses droits et devoirs dans le cadre d'un système particulier de régulation des relations sociales: la démocratie. (Morel, 2000: 47)

Ces éléments, tant en réduction des risques qu'en réduction des méfaits, constituent les obligations de l'État dans une politique en matière de drogues où est recherchée l'augmentation de l'autonomie du citoyen avec une priorité en promotion de la santé.

Il n'y a pas de recette magique. Une politique sociale, quel que soit son domaine, est un processus continu susceptible de s'améliorer, mais également de se détériorer, selon les contingences politiques, sociales et économiques. Pour cela, comme avec toute politique sociale, il faut la suivre, en critiquer les écarts, et rappeler la primauté du bien-être du citoyen, même si ce n'est pas toujours facile. Les mouvements de lutte à la pauvreté ne s'empêchent pas d'exister parce qu'il y aura toujours des pauvres. Ils tentent d'augmenter les bienfaits des politiques gouvernementales et d'en réduire les méfaits. Ils cherchent également à s'assurer que tout n'est pas que discours, mais se traduit sur le terrain par des stratégies concrètes.

La légalisation contrôlée des drogues doit s'inscrire dans une politique gouvernementale qui permette aux citoyens de faire des choix autonomes et éclairés de consommation. Pour ce faire, le gouvernement est responsable de l'environnement dans lequel s'effectuent ces choix et, à cette fin, doit établir des réglementations appropriées. Mais pour identifier ce qui est approprié, encore faut-il avoir une vision claire de l'objectif recherché et des conditions que cela présuppose (Beauchesne, 2006b).

À l'heure actuelle, l'obstacle majeur qui empêche de réfléchir collectivement aux moyens de cette politique publique en matière de drogues est la désinformation de la population soutenue par la prohibition. À cet égard, cet article espère avoir su montrer que des débats peuvent être soulevés dès maintenant en ce qui a trait aux politiques sur les drogues légales, afin éventuellement d'arriver à une politique citoyenne en matière de drogues qui englobe avec cohérence et équité l'ensemble de celles-ci et qui vise la promotion de la santé. 


\section{Références}

Adrian, M., Ferguson, B. S., \& Her, M. (2001). Can alcohol price policies be used to reduce drunk driving? Evidence from Canada. Substance Use $\mathfrak{F}$ Misuse, 36 (13), 1923-1257.

Angell, M. (2004). The Truth about the Drug Companies. Toronto : Random House of Canada Ltd.

Babor, T. F. et al. (2003). Alcohol: No ordinary Commodity - Research and Public Policy. Oxford and London: Oxford University Press.

Beauchesne, L. (2005). Drogues, mythes et dépendance: en parler aux enfants. Montréal: Bayard Canada.

Beauchesne, L. (2006a, C2003). Les drogues: Les coûts cachés de la probibition. Montréal: Bayard Canada.

Beauchesne, L. (2006b) Les drogues: Légalisation et promotion de la santé. Montréal: Bayard Canada.

Brisson, P. (2000). Développement du champ québécois des toxicomanies au $\mathrm{XX}^{\mathrm{e}}$ siècle. In $\mathrm{P}$. Brisson (dir), L'usage des drogues et la toxicomanie (vol. III, 3-44). Boucherville: Gaétan Morin Editeur.

Büchli, D. T. (2002). Révision de la loi fédérale suisse de 1951 sur les stupéfiants et les substances psychotropes. Ottawa: Mémoire déposé au comité sénatorial spécial sur les drogues illicites. Disponible sur le site: <www.parl.gc.ca/ drogues-illicites.asp $>$

Colle, F.-X. (2000). Les drogues en vente libre. Paris: PRAT Éditions, Division de Elsevier Business Information.

Collin, C. (2002). Politique en matière de drogue: Suisse. Ottawa: Rapport préparé pour le Comité spécial du Sénat sur les drogues illicites. Comité sénatorial spécial sur les drogues illicites. Disponible sur le site: <www.parl.gc.ca/ drogues-illicites.asp $>$

Coppel, A. (2002). Peut-on civiliser les drogues? De la guerre à la drogue à la réduction des risques. Paris: La découverte.

Fenn, A. J., \& Schroeter, J. R. (2004). Cigarettes and addiction information: simulating the demand effects of the tobacco industry's 'conspiracy of silence'. Applied Economics, 36, 21-59.

Fisher, M. A. (2003). Physicians and the Pharmaceutical Industry, A Dysfunctional Relationship. Perspectives in Biology and Medecine, 46 (2), 254-272.

Fortin, J. (2006). Privatiser ou non la Société des alcools? Voir au-delà des chiffres. Montréal: UQAM. Disponible sur le site: <www.unites.uqam.ca>.

Garretsen, H. F. L. (2003). The decline of Dutch drug policy? Lessons to be learned. Journal of Substance Use, 8, 2-4.

Garten, S., \& Falkner, V. (2004). Role of mentholated cigarettes in increased nicotine dependence and greater risk of tobacco-attributable disease. Prevention Medicine, 38, 793-798.

Grube, J. W., \& Stewart, K. (2004). Preventing Impaired Driving Using Alcohol Policy. Traffic Injury Prevention, 5, 199-207. 
Haden, M. (2004). Regulation of illegal drugs: an exploration of public health tools. The International Journal of Drug Policy, 15 (4), 225-230.

Hatsukami, D. K., Slade, J., Benowitz, N. L., Biovino, G. A., Gritz, E. R., Leischow, S., \& Warner, K. E. (2001). Reducing tobacco harm: Research challenges and issues. Nicotine $\mathcal{F}$ Tobacco Research, S89-S101.

Jazairi, N. T. (1994). The impact of privatizing the liquor control board of Ontario. York, Toronto: Interim Report, prepared for the Ontario Liquor Boards Employees' Union.

Keizer, B. (2001). La politique sur les drogues des Pays-Bas. Ottawa: Mémoire présenté au Comité spécial du sénat sur les drogues illicites. Disponible sur le site: <www.parl.gc.ca/drogues-illicites.asp>

Keithly, L., Wayne, G. F., Cullen, D. M., \&. Connolly, G. N. (2005). Industry research on the use and effects of levulinic acid: A case study in cigarette additives. Nicotine $\mathbb{\sigma}$ Tobacco Research, 7 (5), 761-771.

Legresley, E., Muggli, M. E., \& Hurt, R. D. (2005). Playing hide-and-seek with the tobacco industry. Nicotine $\mathcal{F}$ Tobacco Research, 7 (1), 27-40.

Levine, H. G., \& Reinarman, C. (2004). Alcobol probibition and drug probibition. Lessons from alcohol policy for drug policy. Amsterdam: CEDRO.

Liberman, J. (2003). Where to for tobacco regulation: time for new approaches? Drug and Alcobol Review, 22, 461-469.

Macdonald, S. (1999). Unrecorded consumption in Ontario, Canada: estimation procedures and research implications. Drug and Alcohol Review, 18 (1), 2129.

Mather, C. (2005). The pipeline and the porcupine: alternate metaphors of the physician-industry relationship. Social Science or Medicine, 60, 1323-1332.

Mitchell, C. N. (1990). The Drug Solution. Ottawa: Carleton University Press.

Morel, A. (2000). Prévenir les toxicomanies. Paris: Dunod.

Muggli, M. E., Hurt, R. D., \& Blanke, D. D. (2003). Science for hire: A tobacco industry strategy to influence public opinion on second hand smoke. Nicotine er Tobacco Research, 5, 303-314.

Muggli, M. E., Hurt, R. D., \& Becker, L. B. (2004a). Turning free speck into corporate speech: Philip Morris' efforts to influence U.S. and European journalists regarding the U.S. EPA report on second hand smoke. Preventive Medicine, 39, 568-580.

Muggli, M. E., Hurt, R. D., \& Repace, J. (2004b). The Tobacco Industry's Political Efforts to Derail the EPA Report on ETS. American Journal of Preventive Medicine, 26 (2), 167-177.

Muggli, M. E., LeGresley, E. M., \& Hurt, R. D. (2004c). Big tobacco is watching: British American Tobacco's surveillance and information concealment at the Guildford depository. The Lancet, 363, 1812-1819.

Nolin, P. C. (2003). Comment réinventer la politique canadienne sur les drogues illicites? Éthique publique, 5 (2), 123-131.

OMS (Organisation mondiale de la santé) (1986). Ottawa: Charte d'Ottawa pour la promotion de la santé. Disponible sur le site: <http://www.euro.who.int/ AboutWHO/Policy/20010827_2?language=French $>$ 
Parascandola, M. (2005). Lessons from the history of tobacco harm reduction: The National Cancer Institute's Smoking and Health Program and the 'less hazardous cigarette'. Nicotine $\mathcal{E}$ Tobacco Research, 7 (5), 779-789.

Robinson, J. (2001). Prescription Games. Toronto: McLelland \& Stewart Ltd.

Room, R. (2004). Alcohol and harm reduction, then and now. Critical Public Health, 14 (4), 329-344.

Santé Canada, Conseil Exécutif Canadien sur les toxicomanies et le Centre canadien de lutte aux toxicomanies (2005). Enquête sur les toxicomanies au Canada, Rapport détaillé, mars.

Statistique Canada (2005 : 8 septembre). Contrôle et vente des boissons alcoolisées. Le Quotidien.

St-Onge, J. C. (2004). L'envers de la pilule. Montréal: Éditions écosociété.

Stockwell, T. (1997). Harm Reduction and Licensed Drinking Settings. In P. G. Erickson, Harm Reduction. A new direction for drug policies and programs (213227). Toronto: University of Toronto Press.

Tracy, J. F. (2004). Between Discourse and Being: The Commodification of Pharmaceuticals in Late Capitalism. The Communication Review, 7, 15-34.

Van Der Smissen, P., \& Picard, J.-M. (1990). Dépénaliser la consommation et le commerce des stupéfiants? L'opinion de juristes. In Le sida, un défi aux droits (177 à 231). Bruxelles: Bruylant.

Warner, K. E. (2002). Tobacco harm reduction: Promise and perils. Nicotine $\mathfrak{E}$ Tobacco Research, S61-S71. 